\title{
Another Train Paradox: May the Myelin Be with You!
}

\author{
Bernard Delalande1, Hirohisa Tamagawa², Vladimir Matveev ${ }^{3}$ \\ ${ }^{1}$ NeuroDynamique.fr, La Verpilliere, France \\ ${ }^{2}$ Department of Mechanical Engineering, Faculty of Engineering, Gifu University, 1-1 Yanagido, Gifu, Japan \\ ${ }^{3}$ Laboratory of Cell Physiology, Institute of Cytology, Russian Academy of Sciences, St. Petersburg, Russia \\ Email: bernard@somasimple.com
}

How to cite this paper: Delalande, B., Tamagawa, H. and Matveev, V. (2021) Another Train Paradox: May the Myelin Be with You! Open Access Library Journal, 8: e7379. https://doi.org/10.4236/oalib.1107379

Received: April 1, 2021

Accepted: May 8, 2021

Published: May 11, 2021

Copyright (C) 2021 by author(s) and Open Access Library Inc.

This work is licensed under the Creative Commons Attribution International License (CC BY 4.0).

http://creativecommons.org/licenses/by/4.0/ (c) (i) Open Access

\begin{abstract}
For more than 70 years, biologists and biophysicists have been trying to unravel the mystery surrounding the saltatory conduction of so-called myelinated neurons. This conduction is indeed very different and faster than for fibres "without" myelin. Many theories have been developed. Albert Einstein used the metaphor of the train to explain the theory of relativity. It is also possible to use a similar metaphor to better understand this transient functioning of the neuron: the transmission of the action potential in myelinated fibres. By studying the various theories that have been put forward and confronting them with physics, mathematics and microscopic anatomical observations, it is possible to refute or confirm certain hypotheses. It is easy and simple, then, to demonstrate unequivocally that the action potential cannot, in any way, jump from node of Ranvier (noR) to node of Ranvier as has been assumed and taught until now. It is possible to describe that the neuron uses an elegant and very simple method to increase the speed of transmission of the neuronal message. It is also important to conclude that this increase in speed, contrary to common belief, has an energetic cost that is greater than expected and that is proportional to the speed and in perfect agreement with the laws of thermodynamics.
\end{abstract}

\section{Subject Areas}

Biophysics, Neuroscience

\section{Keywords}

Axon, Neuron, Saltatory Conduction, Action Potential Propagation, Hodgkin and Huxley Model 


\section{Introduction}

For centuries, scientists have been fascinated by the nervous system and how messages are exchanged and transmitted between the various types of neurons. The nature of the message itself is still controversial because the best known and most widely shared theory, the Hodgkin and Huxley model (HH model), is unable to describe all aspects of the signal. This animal "electricity" has been and remains the subject of intense research and theorising. It can even be said that the mechanism of nerve transmission remains mysterious, and several contradictory hypotheses are still under the spotlight today.

Here are the most common functional theories:

"This nerve impulse propagates electrically" is a common notion among most physiologists [1] [2], while some propose that it is a mechanical soliton [3]. Finally, some attempt to link all the theories to unify them while others return to more plausible bases [4]. Some hypothesise a waveguide [5] of the order of one or more Mhz, but this has so far escaped all our measuring devices and the geometrical configuration imposes a drastic functional limitation.

To complete their explanations and hypotheses, biologists have separated neurons into two categories: the so-called myelinated neurons and those that are not. Of course, neurocytologists do not entirely agree with this last distinction [6]. All axons (with very, very rare exceptions) are surrounded by a myelin sheath. The myelin sheath may be compact and spiralling around the axon or it may be non-compact and form a simple envelope around the axon. The use of the term unmyelinated would suggest that the membrane of some axons is in direct contact with the external environment which would favor the functioning of certain theories.

In recent years, biologists and biophysicists have revived a debate linked to this anatomical distinction: is the propagation of the action potential, for myelinated neurons, saltatory or not?

It is obviously not the purpose of this study to point out all the contradictions that might exist in all the proposed models. The subject remains limited to saltatory conduction and the problems it poses which do not seem to be resolved.

The HH model has always been unable to overcome the contradiction between the position of the AP on the axon and the observed speed. The notion and circulation of local currents, also called electrotonus, seem to be wrong and do not respect the current knowledge about ionic movements in electrolytes.

The solitonic model supported by Heimburg, Jackson and Winlow is much better and proposes to address the mechanical aspects that are totally neglected by the former. The ionic aspect seems to be only a consequence of the wave and this is perhaps one of its weaknesses.

Akaishi's model is based on the known interactions between ions and the forces that can drive them. Unfortunately the computed and observed ionic velocities are lower than required for propagation.

The Jacak's model proposes a propagation based on a waveguide which could 
perhaps answer the problem but the necessary frequencies are beyond anything that has been observed. Occam's razor simply dictates that we discard a theory where the workings have not been verified in practice at this time.

Our approach is very different because we have tried to reconcile the theory with observations on the one hand and physical or mathematical validation on the other. Simple mathematical calculations can, in fact, formally exclude certain hypotheses, even if these are still considered as pillars of biology and biophysics. It is normal to question theories because many branches of science have evolved in parallel with biology, rendering obsolete explanations that were taken for granted. Yesterday's explanations still have their merits, but it is legitimate to find solutions based on current knowledge, while waiting, of course, to be replaced by better theories. Table 1 shows the symbols and definitions used in this study.

\section{A Little Physics in a Short Time}

An observer, Albert, sees a train passing by at a known speed $v$.

He can see it for a duration $t$. The length of the train is

$$
l=v \cdot t
$$

Namely, the train has covered a distance $I$ during this period $t$.

These few sentences may take us back to the algebra problems of our childhood, of course, but they contain the very principles of applied science. The

Table 1. Table of symbols and definitions.

\begin{tabular}{cc}
\hline Symbol & Definition \\
\hline$t_{0}$ & start of the AP spike \\
$t_{1}$ & end of the AP spike and begin of recovery period \\
$t_{2}$ & end of recovery period \\
$d_{A P}$ & action potential duration \\
$d_{\text {spike }}$ & spike duration \\
$l_{\text {myel }}$ & length of the spike for myelinated axons \\
$l_{\text {inoR }}$ & internode length \\
$l_{\text {noR }}$ & node length $=l_{\text {myel }}$ \\
$l_{\text {ummyel }}$ & length of the spike for unmyelinated axons \\
$v_{\text {myel }}$ & conduction velocity for myelinated axon \\
$v_{\text {unmyel }}$ & conduction velocity for unmyelinated axon \\
$d_{t}$ & nistance covered by the AP at $t$ time \\
$n o R_{t}$ & noR reached at $t$ time \\
$n o R$ & nof Ranvier acronym
\end{tabular}


observation of a banal phenomenon, in this case the passage of a train, gives us food for thought. Albert, the observer, applies his knowledge of the speed and duration of an observation to compute the length of the train.

Conversely, he is perfectly able, knowing the duration of an event and the speed of the train, of finding the distance travelled by the train.

The most important conclusion is that if an observer at a given location is able to observe a phenomenon for a specified period of time, then that phenomenon exists at that location and for that specified period of time.

\section{Important Concepts on Neurons}

We will examine the different common and particular properties that make it possible to differentiate neurons.

\subsection{Common Properties for All Neurons}

Considering the electrical component of the action potential (see Figure 1): it has a duration that depends on the type of neuron being considered.

$$
d_{A P}=t_{2}-t_{0}
$$

The duration of the effective signal of the action potential, the spike, is almost constant regardless of the type of neuron considered.

$$
d_{\text {spike }}=t_{1}-t_{0}
$$

There is only one spike at any given time $t$ and at a location $x$ on the axon.

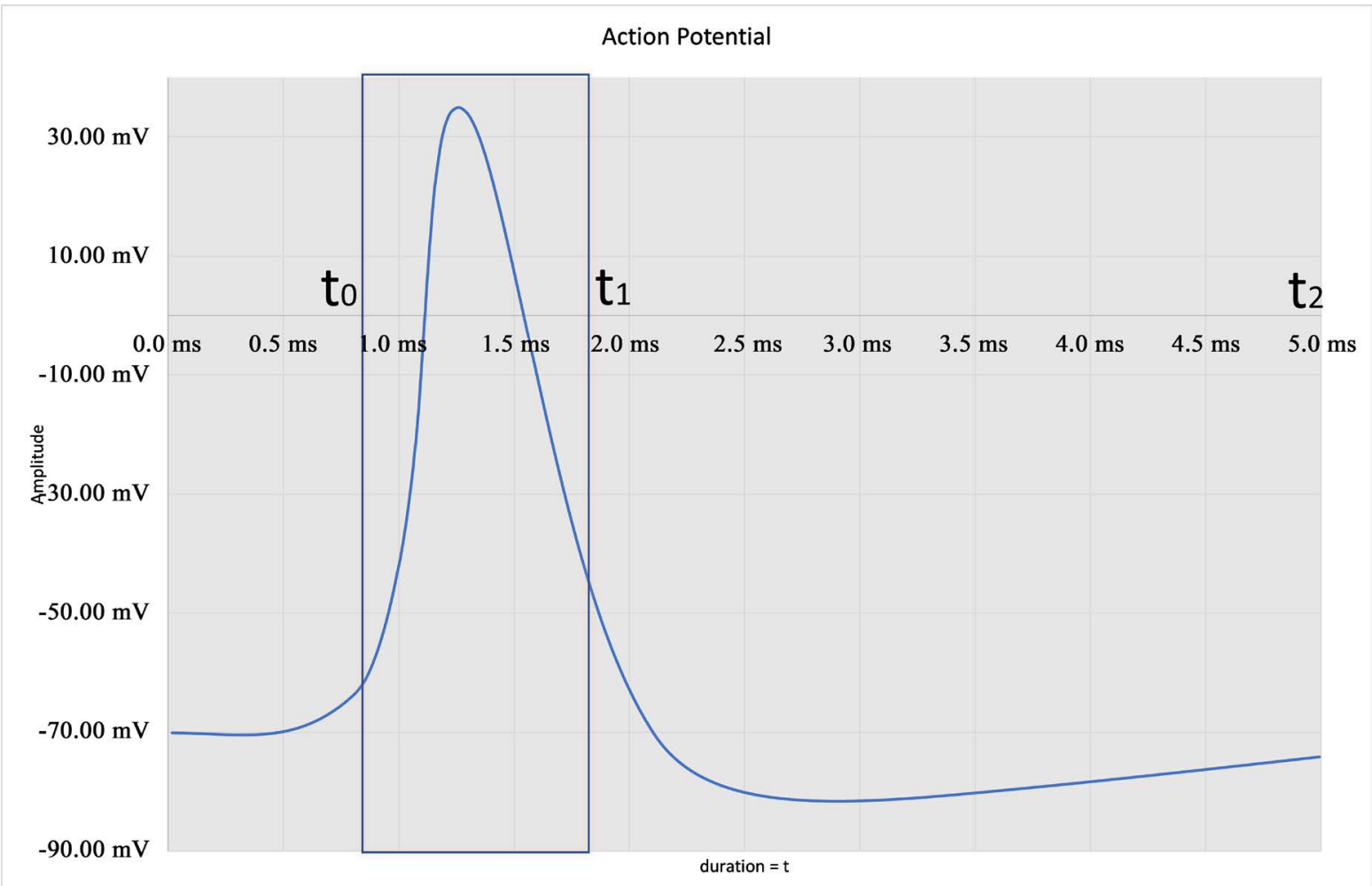

Figure 1. Graphic representation of the electrical component of an action potential. 
This uniqueness suggests that its purely electrical nature may be already questionable. This is particularly true since it is constantly stated that a tiny fraction of the locally available ions are used. What is the phenomenon that prevents most of the unused ions from reacting and neutralizing the action potential at this place?

On the other hand, it is possible to have several action potentials on the same axon and at the same time but of course located at different locations. This primordial and undeniable concept, which has been observed time and again, is not in favor of an electrical propagation.

Each AP is electrically independent of the others that may be present on the axon. Even when there is a pulse train, electrical interference only occurs during the refractory period.

The AP moves (usually) along the axon in only one direction. Its speed of displacement or conduction velocity $v$ depends on the nature of the surrounding myelin.

The conduction velocity is always higher for myelinated neurons. The exception concerning giant axons is very controversial: Most would be a fusion of several hundred of axons, which suggests a very different functioning that may involve neurons selection.

$$
v_{\text {unmyel }}<v_{\text {myel }}
$$

The electrical component of the action potential is incompletely recorded. It does not reflect in any way its spatial dimension related to its geometry. $\mathrm{He}$ is also unable to give us back a major notion, his conduction velocity.

Because it has a duration $d_{\text {spike }}$ and also because it has a velocity ( $v_{\text {myel }}$ or $\left.v_{\text {unmyel }}\right)$, it is possible to say that the spike has a length.

The spike occupies a finite surface on the axon. It can therefore be said that it has a rather cylindrical shape that has a length $l_{\text {myel }}$ or $l_{\text {unmyel }}$.

It should be noted, however, that while the length appears to be proportional to the conduction velocity for unmyelinated fibres, the spike length appears to be fixed for myelinated axons.

This raises more questions about the electrical nature of the AP, a second time.

$$
\begin{gathered}
l_{\text {myel }}=\text { Const } \\
l_{\text {unmyel }}=v_{\text {unmyel }} \cdot d_{\text {spike }}
\end{gathered}
$$

All axons are myelinated [7]. They are all surrounded by specialized cells that produce a myelin barrier that may or may not be compact [8]. It is interesting to ask the question of the existence of so-called unmyelinated fibers since neurocytology dispels this myth through anatomical evidence [6] [9] (Figure 2).

The worst example is the giant squid axon [10] used as a model in the $\mathrm{HH}$ model: It is surrounded by the thickest ( 0.7 to $1.3 \mu \mathrm{m}$ ) myelin layer in the animal kingdom and yet we are taught that it is not myelinated and it is a fusion of multiple axons [11]. 


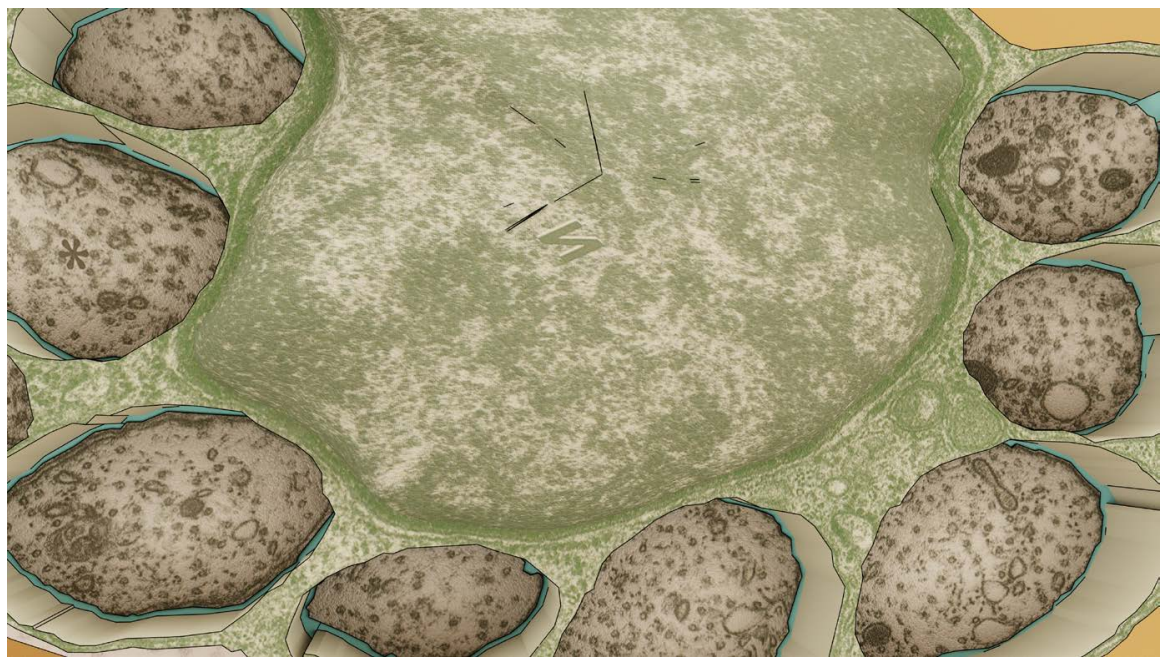

Figure 2. Eight unmyelinated axons surrounded by myelin (in light green). The Schwann nucleus takes the major area. 3D reconstruction from a microscopy by courtesy of Ennio Pannese.

Most of the electrical component of the AP is negative. It is only at the moment of the peak that the voltage becomes very transiently positive before returning to negative again.

\subsection{Unique Properties of the Unmyelinated Neuron}

The propagation of the action potential on the unmyelinated axon is continuously observable.

It is therefore possible to know the position at a time $t$. of the AP by simple computation, with $x_{0}$ the position of the AP at a time $t_{0}$ :

$$
d_{t}=v_{\text {unmyel }} \cdot t+x_{0}
$$

We have seen that for unmyelinated fibers, the action potential has a physical length on the axon. The usual cylindrical shape of the neuron extension allows us to conclude that the AP occupies a surface proportional to the speed.

$$
\begin{gathered}
S=2 \pi \cdot r_{\text {unmyel }} \cdot\left(v_{\text {unmyel }} \cdot d_{\text {spike }}\right) \\
S \propto v_{\text {unmyel }}
\end{gathered}
$$

It is therefore also possible to assume that energy consumption is proportional to speed. It is also likely that the ionic quantities and their counterparts, the ion channels involved, will become too large to ensure any propagation.

It is also perfectly understandable that this operating mode for signal transmission, robust for low speeds, becomes a candidate for failure because it is exposed to possible mechanical obstacles.

The speeds normally observed for this type of neurons are between 0.2 and 3 $\mathrm{m} \cdot \mathrm{s}^{-1}$.

The spike has a more or less constant duration, this length varies between $2 \times$ $10^{-4}$ and $3 \times 10^{-3} \mathrm{~m}$. 


\subsection{Special Properties of the Myelinated Neuron}

In myelinated neurons, conduction is called saltatory although this statement is not yet clear-cut [12] [13] [14] [15]. It is stated, without irrefutable evidence, that the action potential seems to jump from noR to the next one [16] [17]. The process would of course be linked to the presence of myelin, which would improve electrical conduction while reducing energy costs [18].

It can be said that it is not possible to increase the transmission speed of a signal without increasing the energy expenditure. This goes beyond the thermodynamic principles and all the theories we apply and therefore successfully test every day on electrical signals.

We will demonstrate this without any uncertainty or ambiguity.

As mentioned above, the action potential, for myelinated fibers, is observed over a constant length related to the anatomy of the myelin surrounding the axon. This is the size of the noR which is about $1.0 \mu \mathrm{m}$. The small surface area suggests a better robustness.

However, the AP is continuously observable, in time, at this same noR.

On the other hand, the surface area occupied on the axon is greatly reduced and it could therefore be assumed that there is a reduction in the energy and physical resources (ion channels) involved.

This configuration contradicts any possible electrical propagation.

If we know the internode length, it is perfectly possible to know both the distance covered since the instant $t_{0}$ but also the number of jumped nodes noR .

In the same way, we calculate the distance covered, which will then be divided by the length of the internode and the length of the node itself.

Let us not neglect any length because all of them seem to be more crucial than they appear [19] [20] [21].

$$
\begin{gathered}
d_{t}=v_{\text {myel }} \cdot t \\
n o R_{t}=\frac{d_{t}}{l_{\text {inoR }}+l_{\text {noR }}}
\end{gathered}
$$

This gives us with a $l_{\text {inoR }}$ value of $10^{-3}$ and $l_{\text {noR }}$ of $10^{-6} \mathrm{~m}$ and a speed between 4 and $150 \mathrm{~m} \cdot \mathrm{s}^{-1}$ :

This gives already extraordinary numbers for our neurons of 4000 and 149,850 noR jumped in only 1 second.

It is also perfectly possible to state without any doubt with Equation (10) and Equation (11) that the number of noRs crossed is both proportional to the duration $t$ and the conduction speed $v_{\text {myel }}$.

\section{Let's Watch the Trains}

It is possible to solve the mystery of saltatory conduction by using "trains" instead of APs. A single but important distinction is that unlike a real train, the $\mathrm{AP}$, in unmyelinated fibres, does not travel but is rebuilt from place to place. Is it not the case for myelinated fibres? 


\subsection{The Train Metaphor}

Our trains have a length determined by their speed and type. They are more or less long as described above for unmyelinated neurons. A train, on the opposite, appears relatively compressed (it should be even more so) and reflects the length of the AP at a noR (see Figure 3).

The axons will look like rails, of course.

We add tunnels to represent, as it should be, the compact myelin because in fact much of the riddle lies in the darkness of their understanding.

\subsection{An Observer Looking at His Watch}

Our observer is asked to position himself at location X and start his stopwatch as soon as he sees the headlights of a train and stop it when he sees the taillights.

He is certain: the transit time was almost identical for all 3 trains.

He noted that the farthest train seemed to go the fastest. Anyway, faster than the one on the central line.

He has a doubt about the last one: it seems that he appeared directly between the two tunnels and disappeared in the same way. He even had the impression that the train was running well in front of him but more like a movie than a real train, weird.

\subsection{Trains Don't Jump}

Let's twist the neck once and for all to this saltatory conduction.

We have been led to believe for too long that the action potential jumps from noR to noR and that is what explains its increased speed [12] [22].

Let's place a second observer at the exit of the first tunnel and ask him to perform the same measurements at the same time as the first one.

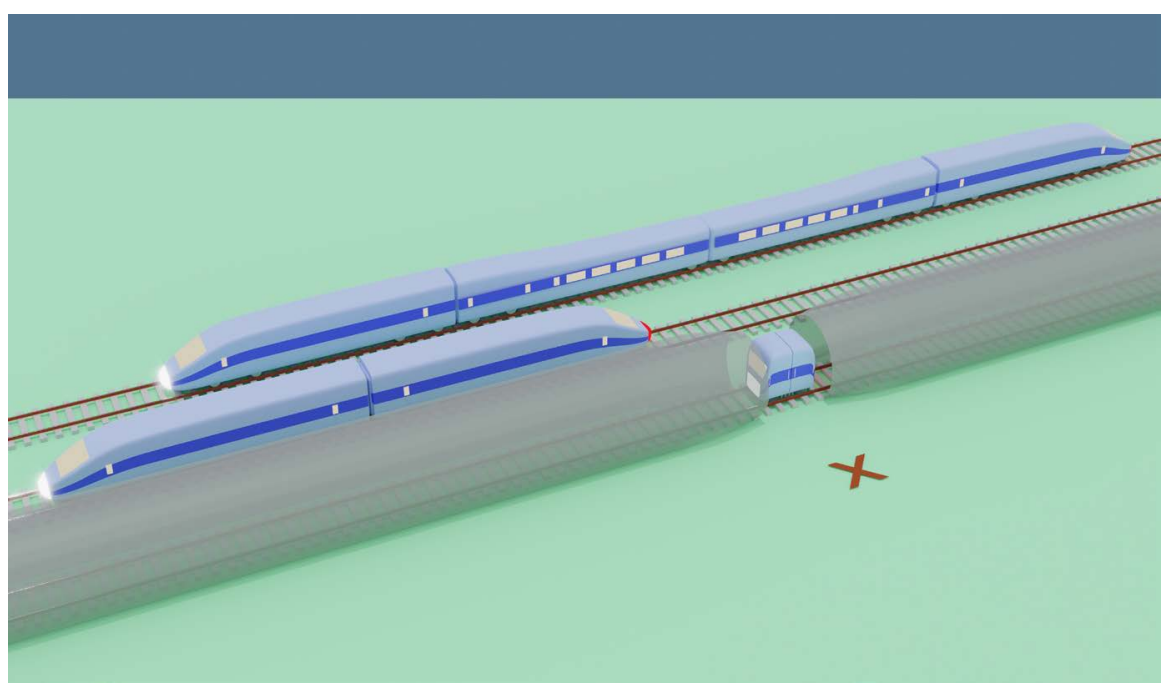

Figure 3. 3 action potentials: Axons are shown as rails. 2 APs on unmyelinated fibers at the top, the fastest is the longer one but its duration is similar. The tunnels are like compact myelin. The AP at noR seems compressed and looks quite stationary. Its duration is the same as the others. 
The second observer must see the train's headlights while the first observer must see the rear lights: there would then be an unequivocal saltatory conduction (see Figure 4).

That is not what is being witnessed [23]!

Otherwise, the observation time should necessarily decrease as the speed increases.

There is only one solution that can satisfy our observation: There are two trains.

It can be said that the number of APs existing on the axon is proportional to the conduction speed (see Figure 5).

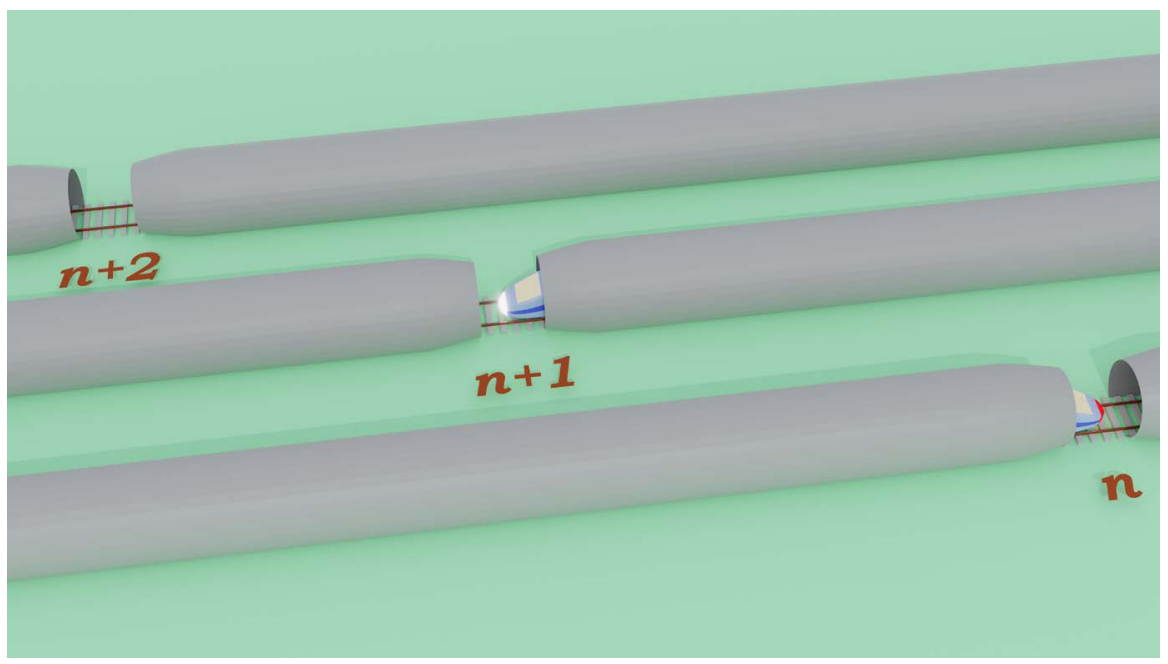

Figure 4. Conventional saltatory conduction: the train jumps at the next node when it ends at the current one.

Numbers of activated nodes vs speed and AP durations

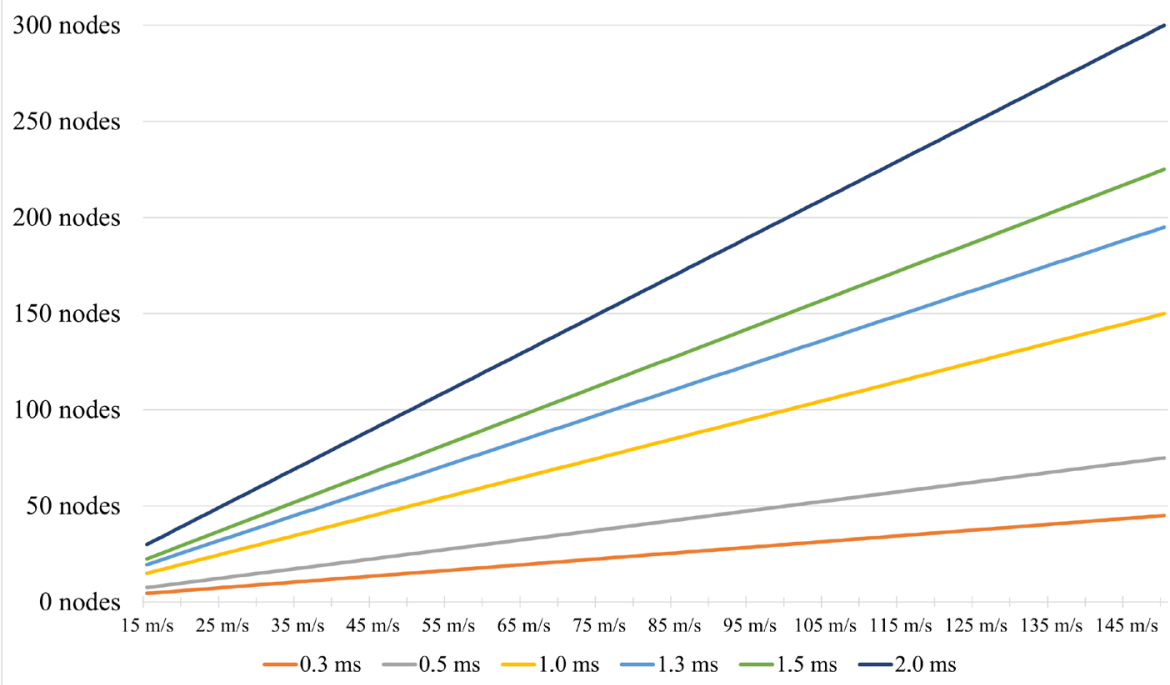

Figure 5. Numbers of noRs activated vs conduction velocity (4 to $150 \mathrm{~m} \cdot \mathrm{s}^{-1}$ ) and internode length, and AP duration ( 0.3 to $2 \mathrm{~ms}$ ). The energetic cost is higher with myelinated neurons. 
We already knew this truth from the beginning of this article with the Equations (1), (10) and (11).

Some of us cannot of course be convinced by this evidence. It doesn't matter because mathematics is always uncompromising.

Let us take an easy and uncontroversial example to satisfy potential critics.

A myelinated fiber with an internode length of $2 \times 10^{-3} \mathrm{~m}$, an AP (spike) of 5 $\times 10^{-4} \mathrm{~s}$ and an average velocity of $40 \mathrm{~m} \cdot \mathrm{s}^{-1}$.

If it is true that it is only at the end of the action potential that the signal jumps to the next noR then we should be able to confirm by computation the speed of $40 \mathrm{~m} \cdot \mathrm{s}^{-1}$.

$$
\frac{1}{d_{\text {spike }}} \cdot l_{\text {inoR }}=\frac{1}{5 \times 10^{-4}} \times 2 \times 10^{-3}=4.0 \mathrm{~m} \cdot \mathrm{s}^{-1} \ll 40 \mathrm{~m} \cdot \mathrm{s}^{-1}
$$

Or by the opposite method of calculation;

$$
v_{\text {myel }} \cdot d_{\text {spike }}=40 \times 5 \times 10^{-4}=2.0 \times 10^{-2} \mathrm{~m} \gg 2 \times 10^{-3} \mathrm{~m}
$$

It is also possible to check that the system reaches its low operating limit of 4.0 $\mathrm{m} \cdot \mathrm{s}^{-1}$ which is well over the length of the internode.

$$
v_{\text {myel }} \cdot d_{\text {spike }}=4.0 \times 5 \times 10^{-4}=2.0 \times 10^{-3} \mathrm{~m}
$$

The system therefore only works when;

$$
v_{\text {myel }} \cdot d_{\text {spike }} \geq l_{\text {inoR }}
$$

It is only logical that it is the front of the train that triggers the departure of the next train. It is unlikely that the rear would be able to trigger an event that would be in front of the head of the train.

Saltatory conduction is not verified but we then have a major new problem: If there are several APs on the axon how, on arrival, there is only one left? Time is the obvious answer! If an AP starts at an noR \#1 then it always ends before the one at noR \#2. There is no possibility of it being transmitted a second time.

\subsection{Electric Trains without Catenary Cables}

It is undeniable that the cells contain ions. It is also not disputed that the movement of these ions creates an electric field and also a potential difference.

It is perfectly possible to elicit an AP with an electrical stimulus, but where is the generator of this impulse in the neuron? A few ions that have changed places, nothing more but nothing less.

Nevertheless, in the absence of an electrical circuit, these movements remain governed by the laws of electrostatics.

Of course, we must try to prove it, whereas an abundance of articles use, for example, an "electric" neuron model, the Hodgkin and Huxley model. It's a kind of reference, but is it accurate?

While it is true, and why contest it, that the action potential is closely linked to the presence of ion channels, it is also perfectly proven that myelin damage, compact or not (as in multiple sclerosis) leads to a slower conduction rate for all 
types of fibres. The action potential is even eliminated when the compact myelin disappears consecutively over a tiny length of the axon [24].

It is therefore also perfectly possible to state that conduction can only be carried out correctly when ion channels are present (which is why unmyelinated fibres are less affected in MS). And, it is undeniably known that the internodal zone has an area where ion channels are completely absent. If the conduction stops then it cannot be electric in the sense that we mean it.

We will explain why the para-nodal zone, on the other hand, has totally essential ion channels.

\subsection{You Shall not Pass!}

It is accepted that the axon membrane undergoes a slight vertical deformation in unmyelinated fibers. We also know that there is no doubt that the membrane undergoes a phase transition during the AP. Nor can we dispute the essential presence of ion channels.

There are also many references [25]-[30] showing that the compression of axons, myelinated or not, leads to a slowing down and then the disappearance of APs.

As it is also known that the optimal conduction rate depends on the quality of the myelin.

We have enough evidence to understand that the basic propagation system is based on a vertical force associated with horizontal translation (see Figure 6). This process is repeated throughout the axon, which obviously wastes time and energy.

If we accept that increased speed is linked to an improvement in the basic system, then we must admit that by reducing the presence of action potential at noR, it leads to a summation of the forces we have described. We have a larger but slower vertical and horizontal component. The solution being of course to transform this large quantity gathered at the noR into a faster horizontal component than usual.

The best way to change the speed of a system that is too slow is to replace it with a faster one.

If the neuron membrane is prevented from deforming by surrounding it with a sufficiently rigid envelope made of layers, reinforced by tight junctions, the mechanical wave is then sent to the next node in a liquid medium without much loss.

And it works: We use this kind of device every day in the industry.

It is quite easy to verify because the available speed can go up to more than $1500 \mathrm{~m} \cdot \mathrm{s}^{-1}$.

We need to solve our last problem.

The AP that activates the next noR is not transmitted because the ion channels under the myelin activate as soon as the "liquid" wave passes. These firm locks provide better strength and pressure transmission and limit ionic movements at the noR (see Figure 6). 


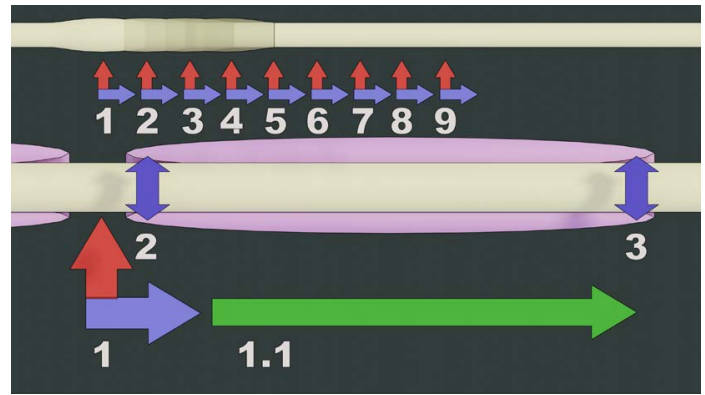

Figure 6. Propagation methods of APs in unmyelinated vs myelinated neurons.

It becomes clear that the minimal functional unit of the nervous system is not, and has never been, the neuron alone.

It is always associated with myelin and cannot function without it.

It is therefore possible to state that there are several APs on the myelinated axon located, in sequence, at the nodes of Ranvier. It is therefore mandatory that they are electrically isolated. It is impossible for two consecutive APs, whose speed is very slow, to be electrically linked with an electrical phenomenon of higher speed.

This paradox is only solved if the APs are electrically insulated (the electromagnetic and electric fields persist even in the absence of an electric circuit).

\section{Conclusions}

We have shown that conduction is not saltatory as some people thought. We provide mathematical evidence that can be verified but is not questionable and confirm our theory, which sheds new light on events that seemed obscure and complex.

This does not detract from the precursors who have tried to give a "truth" but which is too incomplete, limited to a single aspect but pleasant.

We provide an elegant and straightforward solution that better explains the facts observed.

This vision, which is based on scientific observations, restores the nobility of this essential couple of the nervous system: the neuron and its companion, the myelin.

\section{Conflicts of Interest}

The authors declare no conflicts of interest regarding the publication of this paper.

\section{References}

[1] Hodgkin, A.L. (1937) Evidence for Electrical Transmission in Nerve: Part I. The Journal of Physiology, 90, 183-210. https://doi.org/10.1113/jphysiol.1937.sp003507

[2] Hodgkin, A.L. (1937) Evidence for Electrical Transmission in Nerve: Part II. The Journal of Physiology, 90, 211-232. https://doi.org/10.1113/jphysiol.1937.sp003508

[3] Heimburg, T. and Jackson, A.D. (2005) On Soliton Propagation in Biomembranes 
and Nerves. Proceedings of the National Academy of Sciences of the United States of America, 102, 9790-9795. https://doi.org/10.1073/pnas.0503823102

[4] Akaishi, T. (2018) Saltatory Conduction as an Electrostatic Compressional Wave in the Axoplasm. The Tohoku Journal of Experimental Medicine, 244, 151-161. https://doi.org/10.1620/tjem.244.151

[5] Jacak, J.E. and Jacak, W.A. (2020) New Wave-Type Mechanism of Salutatory Conduction in Myelinated Axons and Micro-Salutatory Conduction in C Fibres. European Biophysics Journal, 49, 343-360. https://doi.org/10.1007/s00249-020-01442-Z

[6] Pannese, E. (1994) Neurocytology: Fine Structure of Neurons, Nerve Processes, and Neuroglial Cells. G. Thieme Verlag, Stuttgart; Thieme Medical Publishers, New York.

[7] De Pittà, M. (2019) Myelin and Saltatory Conduction. arXiv: 1708.00534. http://arxiv.org/abs/1708.00534

[8] Bear, R.S., Schmitt, F.O. and Young, J.Z. (1937) The Sheath Components of the Giant Nerve Fibres of the Squid. Proceedings of the Royal Society of London. Series B, Biological Sciences, 123, 496-504. https://doi.org/10.1098/rspb.1937.0065

[9] Fraher, J.P. and O’Sullivan, A.W. (2000) Interspecies Variation in Axon-Myelin Relationships. Cells Tissues Organs, 167, 206-213. https://doi.org/10.1159/000016783

[10] Brown, E.R. and Abbott, N.J. (1993) Ultrastructure and Permeability of the Schwann Cell Layer Surrounding the Giant Axon of the Squid. Journal of Neurocytology, 22, 283-298. https://doi.org/10.1007/BF01187127

[11] Martin, R. (1965) On the Structure and Embryonic Development of the Giant Fibre System of the Squid Loligo vulgaris. Zeitschrift für Zellforschung und Mikroskopische Anatomie, 67, 77-85. https://doi.org/10.1007/BF00339277

[12] Fitzhugh, R. (1962) Computation of Impulse Initiation and Saltatory Conduction in a Myelinated Nerve Fiber. Biophysical Journal, 2, 11-21.

https://doi.org/10.1016/S0006-3495(62)86837-4

[13] Giuliodori, M.J. and DiCarlo, S.E. (2004) Myelinated vs. Unmyelinated Nerve Conduction: A Novel Way of Understanding the Mechanisms. Advances in Physiology Education, 28, 80-81. https://doi.org/10.1152/advan.00045.2003

[14] Laporte, Y. (1951) Continuous Conduction of Impulses in Peripheral Myelinated Nerve Fibers. The Journal of General Physiology, 35, 343-360. https://doi.org/10.1085/jgp.35.2.343

[15] Rasminsky, M. and Sears, T.A. (1972) Internodal Conduction in Undissected Demyelinated Nerve Fibres. The Journal of Physiology, 227, 323-350.

https://doi.org/10.1113/jphysiol.1972.sp010035

[16] Huxley, A.F. and Stampfli, R. (1951) Effect of Potassium and Sodium on Resting and Action Potentials of Single Myelinated Nerve Fibers. The Journal of Physiology, 112, 496-508. https://doi.org/10.1113/jphysiol.1951.sp004546

[17] Huxley, A.F. and Stämpfli, R. (1949) Evidence for Saltatory Conduction in Peripheral Myelinated Nerve Fibres. The Journal of Physiology, 108, 315-339. https://doi.org/10.1113/jphysiol.1949.sp004335

[18] Sangrey, T. and Levy, W.B. (2005) Conduction Velocity Costs Energy. Neurocomputing, 65-66, 907-913. https://doi.org/10.1016/j.neucom.2004.10.091

[19] Ford, M.C., Alexandrova, O., Cossell, L., Stange-Marten, A., Sinclair, J., KoppScheinpflug, C., et al. (2015) Tuning of Ranvier Node and Internode Properties in Myelinated Axons to Adjust Action Potential Timing. Nature Communications, 6, Article No. 8073. https://doi.org/10.1038/ncomms9073

[20] Foust, A., Popovic, M., Zecevic, D. and McCormick, D.A. (2010) Action Potentials 
Initiate in the Axon Initial Segment and Propagate through Axon Collaterals Reliably in Cerebellar Purkinje Neurons. Journal of Neuroscience, 30, 6891-6902. https://doi.org/10.1523/JNEUROSCI.0552-10.2010

[21] Foust, A.J., Yu, Y., Popovic, M., Zecevic, D. and McCormick, D.A. (2011) Somatic Membrane Potential and Kv1 Channels Control Spike Repolarization in Cortical Axon Collaterals and Presynaptic Boutons. Journal of Neuroscience, 31, 15490 15498. https://doi.org/10.1523/JNEUROSCI.2752-11.2011

[22] Goodman, B.E. and Waller, S.B. (2002) Propagation of Action Potentials in Myelinated vs. Unmyelinated Neurons. Advances in Physiology Education, 26, 223. https://doi.org/10.1152/advan.00023.2002

[23] Debanne, D., Campanac, E., Bialowas, A., Carlier, E. and Alcaraz, G. (2011) Axon Physiology. Physiological Reviews, 91, 555-602.

https://doi.org/10.1152/physrev.00048.2009

[24] Baraban, M., Mensch, S. and Lyons, D.A. (2016) Adaptive Myelination from Fish to Man. Brain Research, 1641, 149-161. https://doi.org/10.1016/j.brainres.2015.10.026

[25] Court, F.A., Sherman, D.L., Pratt, T., Garry, E.M., Ribchester, R.R., Cottrell, D.F., et al. (2004) Restricted Growth of Schwann Cells Lacking Cajal Bands Slows Conduction in Myelinated Nerves. Nature, 431, 191-195.

https://doi.org/10.1038/nature02841

[26] Fern, R. and Harrison, P.J. (1994) The Relationship between Ischaemic Conduction Failure and Conduction Velocity in Cat Myelinated Axons. Experimental Physiology, 79, 571-581. https://doi.org/10.1113/expphysiol.1994.sp003790

[27] Ffrench-Constant, C., Colognato, H. and Franklin, R.J.M. (2004) Neuroscience. The Mysteries of Myelin Unwrapped. Science, 304, 688-689. https://doi.org/10.1126/science.1097851

[28] Grandis, M., Leandri, M., Vigo, T., Cilli, M., Sereda, M.W., Gherardi, G., et al. (2004) Early Abnormalities in Sciatic Nerve Function and Structure in a Rat Model of Charcot-Marie-Tooth Type 1A Disease. Experimental Neurology, 190, 213-223. https://doi.org/10.1016/j.expneurol.2004.07.008

[29] Padrón, R. and Mateu, L. (1982) Repetitive Propagation of Action Potentials Destabilizes the Structure of the Myelin Sheath. A Dynamic X-Ray Diffraction Study. Biophysical Journal, 39, 183-188. https://doi.org/10.1016/S0006-3495(82)84506-2

[30] Williams, A. C. and Brophy, P.J. (2002) The Function of the Periaxin Gene during Nerve Repair in a Model of CMT4F. Journal of Anatomy, 200, 323-330. https://doi.org/10.1046/j.1469-7580.2002.00038.x 